\title{
Comparison of bispectral index and entropy monitoring in patients undergoing internalisation of deep brain stimulators
}

\author{
Suparna Bharadwaj, Mahesh Nagappa, Audrey Tan, Jigesh Mehta, Pirjo Manninen, \\ Lashmi Venkatraghavan
}

\begin{abstract}
Introduction: Depth of anaesthesia (DOA) monitors are shown to reduce the intra-operative dose of anaesthetic agents, provide haemodynamic stability and shorten emergence times. Electroencephalography (EEG) based DOA monitors such as bispectral index (BIS) and entropy have been calibrated and validated in healthy subjects. Hence the clinical effectiveness of these monitors may be affected when monitoring patients with neurological disorders (e.g., epilepsy, dystonia, dementia and Parkinson's disease).The aim of this study was to determine whether BIS and entropy correlate with each other and with clinical indices of DOA in patients with movement disorders under general anaesthesia (GA). Materials and Methods: We conducted a prospective, observational study in patients with movement disorders undergoing internalization of deep brain stimulators. All patients received standard GA with age-adjusted mean alveolar concentration (aaMAC) of an inhalational agent between 0.7 and I.I. BIS and entropy sensors were applied on the patient's left forehead. Data collected included clinical parameters and EEG-based DOA indices. Correlation analysis was performed between entropy, BIS and the clinical indices of DOA. Bland Altman analysis was performed to determine the agreement between BIS and entropy. Results: Thirty patients were studied (mean age was $58.4 \pm$ II years, male: female 18:12 and weight $79.2 \pm 17 \mathrm{~kg}$ ). Indications for deep brain stimulation were Parkinson's disease $(n=25)$, essential tremors $(n=2)$ and dystonia $(n=3)$. There was a very strong positive correlation between BIS and response entropy (RE) $(r=0.932)$ and BIS and state entropy (SE) $(r=0.950)$ and a strong negative correlation among aaMAC and BIS, RE and SE with $r$ values of -0.686 , -0.788 and -0.732 , respectively. However, there was no correlation between BIS, RE, SE and haemodynamic values. Conclusion: Our study showed that BIS and entropy perform well in patients with movement disorders. There was a good correlation between the BIS and entropy devices. Haemodynamic parameters were not reliable indicators of DOA in these patients.
\end{abstract}

Key words: Bispectral index, deep brain stimulator, entropy, movement disorders, Parkinson's disease

Department of Anaesthesia and Critical Care, Toronto

Western Hospital, University Health Network, University of Toronto, Toronto, Ontario, Canada

Address for correspondence:

Dr. Lashmi Venkatraghavan, Department of Anaesthesia, Toronto Western Hospital, 399 Bathurst Street, Toronto, Ontario M5T 2S8, Canada. E-mail: lashmi.venkatraghavan@uhn.ca

\begin{tabular}{|l|l|}
\hline \multicolumn{2}{|c|}{ Access this article online } \\
\hline Quick Response Code: & Website: \\
\hline & www.jnaccjournal.org \\
\cline { 1 - 2 } & \\
\hline & \\
\hline
\end{tabular}

\section{INTRODUCTION}

Monitoring the depth of anaesthesia (DOA) using processed electroencephalography (EEG) has been shown to reduce intra-operative awareness. ${ }^{[1]}$ They

This is an open access article distributed under the terms of the Creative Commons Attribution-NonCommercial-ShareAlike 3.0 License, which allows others to remix, tweak, and build upon the work non-commercially, as long as the author is credited and the new creations are licensed under the identical terms.

For reprints contact: reprints@medknow.com

How to cite this article: Bharadwaj S, Nagappa M, Tan A, Mehta J, Manninen P, Venkatraghavan L. Comparison of bispectral index and entropy monitoring in patients undergoing internalisation of deep brain stimulators. J Neuroanaesthesiol Crit Care 2016;3:25-32. 
also have been shown to reduce the intra-operative dose of anaesthetic agents and vasopressors as well as provide haemodynamic stability and shortened emergence times. ${ }^{[2-5]}$ The use of DOA monitors has been recommended for elderly patients who are at risk for post-operative adverse outcomes. ${ }^{[6]}$ The bispectral index (BIS), (Aspect Medical Systems, Newton, MA, USA) and the entropy (GE Healthcare, Datex Ohmeda Division, Helsinki, Finland) are the commonly used DOA monitors derived from processed EEG. ${ }^{[7]}$ The BIS combines the power spectral analysis with bispectral analysis between the component frequencies of the EEG signal. ${ }^{[2]}$ Entropy analyses the amount of disorder in the EEG signals ("state" entropy [SE]) and as the EEG signals become more regular during anaesthesia, this results in decreased entropy. It also measures the irregularity of the frontalis electromyogram, which diminishes as anaesthesia deepens providing an indication of muscle activity ("response" entropy [RE]). The RE index ranges from 0 to 100, whereas the SE index spans from 0 to $91 .{ }^{[8]}$

The calibrations of these EEG-based monitors were originally performed on subjects with normal cerebral function. ${ }^{[9]}$ The algorithm for BIS was derived from a database of predominantly fit, healthy adult patients and volunteers. ${ }^{[10]}$ Hence, the clinical effectiveness of these monitors may be affected when monitoring patients with neurological disorders (e.g., epilepsy, dystonia, dementia and Parkinson's disease) and in patients taking psychoactive medications. ${ }^{[11,12]}$ These patients may also have a large variation in inter-individual response to anaesthetic agents and sensitivity to the anaesthesia. ${ }^{[13]}$ Seizure activity has also been shown to cause interference with these measurements. ${ }^{[14,15]}$ Under these circumstances, the EEG activity may be altered leading to invalid BIS or entropy readings. ${ }^{[16,17]}$ The EEG-based DOA monitors have not been validated in these subsets of the population. There are only anecdotal observations of variability in the applicability of EEG-derived DOA monitors in patients with neurological disorders and psycho-pharmacotherapy. ${ }^{[18,19]}$

Deep brain stimulation (DBS) is an increasingly popular treatment for patients with movement disorders such as Parkinson's disease and dystonia. Surgery for DBS is usually performed in two stages. The first stage consists of accurate localisation of the target nuclei and insertion of the DBS electrodes and is often performed with an awake patient. The second stage, the internalisation of the electrodes and insertion of a pulse generator is usually performed under general anaesthesia (GA). The aim of our study was to determine whether EEG-based DOA monitors, BIS and entropy, correlate with each other and with clinical indices of DOA namely heart rate (HR), blood pressure (BP) and age-adjusted mean alveolar concentration (aaMAC) of inhalational anaesthetic agents, in patients with movement disorders.
We hypothesized that there would be correlation among BIS, entropy and the clinical indices of DOA in patients with movement disorders undergoing GA for internalization of DBS electrodes.

\section{MATERIALS AND METHODS}

\section{Study design}

After institutional ethics board approval and written informed patient consent, we conducted a prospective observational study in patients with movement disorders undergoing internalisation of DBS under GA.

\section{Participants}

Consecutive adult patients ( $>18$ years of age) with movement disorders (Parkinson's disease, dystonia, essential tremors) who were scheduled for elective internalization of DBS electrodes under GA were enrolled for the study. Patients undergoing DBS insertion for other indications (depression, obsessive-compulsive disorder or pain) were excluded from the study.

\section{Anaesthetic management}

All study patients had their DBS electrodes inserted 24-48 $\mathrm{h}$ prior to internalisation. They were maintained on all their regular medications on the day of surgery. In the operating room, routine standard monitors were applied including five-lead electrocardiogram, non-invasive $\mathrm{BP}$, pulse oximetry, capnography, temperature and age-adjusted end-tidal anaesthetic agent monitoring. GA was induced with intravenous (IV) propofol (1-3 mg/kg) and fentanyl (1-2 mcg/ $\mathrm{kg}$ ) and IV rocuronium $(0.6 \mathrm{mg} /$ $\mathrm{kg}$ ) for tracheal intubation. Anaesthesia was maintained with oxygen, air, sevoflurane or desflurane titrated to aaMAC between 0.7 and 1.1. End-tidal $\mathrm{CO}_{2}$ was maintained at normal values. Prophylactic antibiotics (cefazolin or clindamycin) and antiemetics (ondansetron [4 mg] or dexamethasone [8 mg]) were administered as per our routine practice. Intra-operatively, patients received bolus doses of IV fentanyl (25 mcg) or propofol (20-50 mg) for stimulating parts of the surgery. Intra-operative haemodynamics (BP and HR) were maintained within $20 \%$ of baseline using IV fluids, vasopressors and additional anaesthesia as needed. After the closure of the skin incisions, the inhalational agent was turned off and the neuromuscular blockade was reversed with IV neostigmine $0.05 \mathrm{mg} /$ $\mathrm{kg}$ and glycopyrrolate $0.01 \mathrm{mg} / \mathrm{kg}$. Tracheal extubation was performed when the patients met extubation criteria. Patients were then transferred to post-operative care unit, and standard post-operative care was carried out.

\section{Electroencephalography acquisition}

A Datex-Ohmeda S / 5 M-ENTROPY Module (GE Healthcare, Datex-Ohmeda Division, Helsinki, Finland) with a GE three electrode entropy sensor was used for monitoring entropy. A standalone BIS monitor (Aspect Medical Systems, Newton, MA, USA) along with 
a four-electrode BIS Quatro Sensor was used for monitoring BIS. The BIS smoothing time was set at $15 \mathrm{~s}$. The electrodes sensors were applied according to manufacturer's instructions prior to induction of anaesthesia. Both sensors were placed using the left temporal-frontal montage, one above the other in a random sequence. The anaesthesiologists caring for the patient were aware of the BIS and entropy values, but the anaesthetic management was targeted only to the clinical endpoints (BP, HR).

\section{Data sources/measurement}

Data collected include patient demographics, medical comorbidities, anaesthetic management, total drug doses and adverse events. Haemodynamic values (HR, mean arterial BP [MAP]), aaMAC, BIS and entropy data were recorded at following surgical time points: T1 - awake (pre-induction), T2 - loss of response to speech, T3 - loss of eyelash reflex, T4 - intubation, T5 - knife to skin, T6 - stable anaesthetic maintenance (prior to tunneling in neck), T7 - tunneling in the neck, T8 - start of skin closure, T9 - end of skin closure, T10 - anaesthetic agent turned off, T11 - obeying commands, T12 - extubation and T13 - post-extubation [Table 1]. ${ }^{[20]}$ At $24 \mathrm{~h}$ after surgery patients were interviewed and asked to fill in a modified Brice questionnaire to assess for intra-operative awareness [Appendix 1]. ${ }^{[21]}$

\section{Statistical analysis}

The primary outcome of the study was to determine the correlation between EEG indices (BIS, SE, RE) and the clinical indices (HR, MAP, aaMAC) of DOA at the different surgical time points in patients with movement disorders. The secondary outcome was to examine the agreement between BIS and entropy. Spearman and Pearson correlation analysis was performed between RE/SE and BIS and also with haemodynamics (HR and MAP) and aaMAC of the inhalational anaesthetic agent. Band-Altman analysis was performed to determine the agreement between BIS and entropy (RE and SE). We chose a sample size of 30 patients based on the previous observational studies correlating EEG based DOA monitors and clinical parameters. ${ }^{[20,2]}$ Data were presented as mean \pm standard deviation for continuous variables and percentage for categorical variables. Continuous variables were tested using paired $t$-test. Two-tailed $P<0.05$ was considered to be significant. Statistical analysis was done usingSPSS statistical software version 18 (SPSS Inc., Chicago, IL, USA) and GraphPad Prism version 6.00 (GraphPad Software, San Diego, California, USA).

\section{RESULTS}

Thirty-six consecutive patients were screened for eligibility and 30 patients were subsequently enrolled. Of the 6 patients excluded, 3 patients refused consent to participate in the study; 3 patients had both the insertion and internalisation of DBS electrodes under GA. The demographic data are as shown in Table 2. The numerical and graphical trends of RE, SE, BIS, HR, MAP and aaMAC at different surgical time points (T1-T13) are shown in Table 3 and Figure 1. There was a progressive reduction in SE, RE and BIS values from pre-induction (T1) till surgical incision (T5) and then the values plateaued

\section{Table 1: Study time points. Modified from Paolo Martorano et al}

\begin{tabular}{ll}
\hline Times & Anesthetic steps \\
\hline T1 & Awake (preinduction) \\
T2 & Loss of response to speech \\
T3 & Loss of eyelash reflex \\
T4 & Intubation \\
T5 & Knife to skin \\
T6 & Maintenance (prior to tunneling) \\
T7 & Tunneling in the neck \\
T8 & Skin closure start \\
T9 & Skin closure end \\
T10 & Anesthetic agent turned off \\
T11 & Obeying commands \\
T12 & Extubation \\
T13 & Postextubation \\
\hline
\end{tabular}

Table 2: Demographic data

\begin{tabular}{lc}
\hline Patient characteristics & \\
Age $(\text { year })^{*}$ & $58.4 \pm 11$ \\
Male $n(\%)$ : Female $n(\%)$ & $18(60 \%): 12(40 \%)$ \\
Weight $(\mathrm{kg})^{*}$ & $79.2 \pm 17$ \\
Height $(\mathrm{cm})^{*}$ & $169.2 \pm 10$ \\
Duration $(\mathrm{min})^{*}$ & $80.9 \pm 13$ \\
ASA-2 $n(\%)$ & $8(27 \%)$ \\
ASA-3 $n(\%)$ & $22(73 \%)$ \\
Conditions $n(\%)$ & \\
Parkinsonism & $25(83.3)$ \\
Essential tremors & $2(6.7)$ \\
Dystonia depression & $3(10)$ \\
Anesthetic agents & \\
Propofol $(\mathrm{mg})^{* *}$ & $183 \pm 66$ \\
Fentanyl $(\mu \mathrm{g})^{* *}$ & $103 \pm 38$ \\
Midazolam $(\mathrm{mg})$ & $0.3 \pm 0.6$ \\
Rocuronium $(\mathrm{mg})^{* *}$ & $40 \pm 8$ \\
Desflurane $n(\%)$ & $24(80)$ \\
Sevoflurane $n(\%)$ & $6(20)$ \\
\hline
\end{tabular}

*Values are presented as (mean \pm SD), **Total intra-operative dose. $\mathrm{SD}=$ Standard deviation 


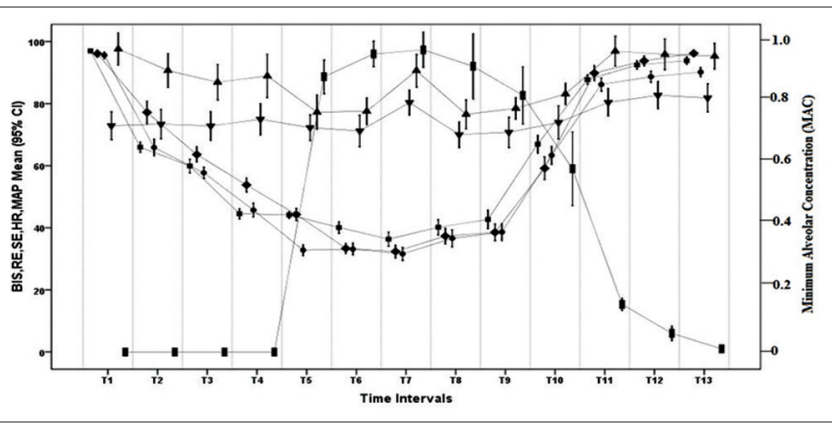

Figure 1: Error bar graph showing the trend of bispectral index, response entropy, state entropy, heart rate, mean arterial blood pressure and age-adjusted mean alveolar concentration in the secondary axis (right) during the various time intervals (T1-T13). T1 - awake (pre-induction), T2 - loss of response to speech, T3 - loss of eyelash reflex, T4 - intubation, T5 - knife to skin, T6 - stable anaesthetic maintenance (prior to tunneling in neck), T7 - tunneling in the neck, T8 - start of skin closure, T9 - end of skin closure, T10 - anaesthetic agent turned off, T11 - obeying commands, T12 - extubation and T13 - post-extubation

\begin{tabular}{ll} 
& Bispectral index (BIS) \\
& Response entropy (RE) \\
& State entropy (SE) \\
& Minimum alveolar concentration (MAC) \\
& Meart rate (HR) \\
\hline
\end{tabular}

until the surgical closure (T9). All three indices showed an increasing trend from T9 (end of skin closure) until post-extubation (T13). The MAP and HR also showed a similar trend in relation to various surgical time points except during the tunneling of the neck (T7) when there was an increase in HR and MAP values. The aaMAC values showed an opposite trend with a progressive increase from $\mathrm{T} 1$ to $\mathrm{T} 5$ and decreasing trend from $\mathrm{T} 9$ to T13 with plateauing in between.

\section{Correlation analysis}

Correlation analysis showed a very strong positive correlation between BIS and RE $(r=0.932)$ and BIS and SE $(r=0.950)$ and a strong negative correlation between aaMAC and BIS, RE and SE with $r$ values of $-0.686,-0.788,-0.732$, respectively. However, there were no correlations between BIS, RE, SE and the haemodynamic values (HR and MAP) [Table 4 and Figures 2 and 3].

\section{Analysis of agreement}

In total, 390 data points for each parameter were used for Bland-Altman analysis [Figure 4]. The bias (or difference) in the relationship between BIS versus $R E$ and $S E$ is illustrated in Figure 4 where the difference between BIS and RE/SE is plotted against the average (BIS and RE/SE) of the values. In Band-Altman analysis, the bias was less with the RE (-0.3; level of bias almost equal to zero;
Table 3: The trends of RE, SE, BIS, HR, MAP, and aaMAC at different study time points

\begin{tabular}{lcccccc}
\hline Time & BIS & RE & SE & $\begin{array}{c}\text { Heart rate } \\
\text { (per minute) }\end{array}$ & $\begin{array}{c}\text { Mean } \\
\text { arterial } \\
\text { pressure } \\
\text { (mm Hg) }\end{array}$ \\
& & & & \multicolumn{3}{c}{$\begin{array}{c}\text { aaMAC } \\
\text { (mm }\end{array}$} \\
& & & & $97 \pm 13$ & 0 \\
T1 & $96 \pm 1$ & $96 \pm 3$ & $95 \pm 2$ & $72 \pm 11$ & $90 \pm 14$ & 0 \\
T2 & $65 \pm 4$ & $77 \pm 9$ & $65 \pm 7$ & $73 \pm 12$ & $86 \pm 15$ & 0 \\
T3 & $59 \pm 5$ & $63 \pm 6$ & $57 \pm 4$ & $72 \pm 12$ & $88 \pm 18$ & 0 \\
T4 & $44 \pm 4$ & $53 \pm 5$ & $45 \pm 5$ & $74 \pm 13$ & $77 \pm 14$ & $0.88 \pm 0.14$ \\
T5 & $44 \pm 2$ & $44 \pm 5$ & $32 \pm 4$ & $72 \pm 11$ & $77 \pm 11$ & $0.96 \pm 0.11$ \\
T6 & $40 \pm 4$ & $33 \pm 4$ & $33 \pm 5$ & $71 \pm 13$ & $90 \pm 14$ & $0.97 \pm 0.15$ \\
T7 & $36 \pm 6$ & $32 \pm 5$ & $31 \pm 5$ & $80 \pm 10$ & $76 \pm 12$ & $0.92 \pm 0.27$ \\
T8 & $40 \pm 6$ & $37 \pm 6$ & $36 \pm 7$ & $70 \pm 11$ & $78 \pm 9$ & $0.82 \pm 0.24$ \\
T9 & $42 \pm 8$ & $49 \pm 9$ & $38 \pm 7$ & $70 \pm 13$ & $83 \pm 9$ & $0.59 \pm 0.31$ \\
T10 & $66 \pm 7$ & $59 \pm 6$ & $63 \pm 7$ & $73 \pm 14$ & $96 \pm 12$ & $0.15 \pm 0.05$ \\
T11 & $87 \pm 3$ & $89 \pm 6$ & $86 \pm 5$ & $80 \pm 11$ & $95 \pm 13$ & $0.06 \pm 0.06$ \\
T12 & $92 \pm 3$ & $93 \pm 4$ & $88 \pm 4$ & $82 \pm 11$ & $95 \pm 11$ & $0.01 \pm 0.03$ \\
T13 & $93 \pm 3$ & $96 \pm 2$ & $90 \pm 3$ & $81 \pm 12$ & &
\end{tabular}

Values are presented as mean \pm SD. RE=Response entropy, $\mathrm{SE}=$ State entropy, BIS=Bispectral index, $\mathrm{HR}=$ Heart rate, $\mathrm{MAP}=$ Mean arterial pressure, aaMAC $=$ Age-adjusted minimum alveolar concentration, $\mathrm{T} 1=$ Awake (pre-induction), T2=Loss of response to speech, T3=Loss of eyelash reflex, $\mathrm{T} 4=$ Intubation, $\mathrm{T} 5=\mathrm{Knife}$ to skin, T6=Stable anaesthetic maintenance, T7=Tunneling in the neck, T8=Start of skin closure, T9=End of skin closure, T10=Anaesthetic agent turned off, T11=Obeying commands, T12=Extubation and T13=Post-extubation, $\mathrm{SD}=$ Standard deviation

one sample $t$-test $P=0.526)$ than the SE (3.5; one sample $t$-test $P=0.00$ ) and the bias was more widely distributed in RE (confidence interval $=-17.9-17.3$ ) than SE (confidence interval $=-11.2-18.3$ ). There was a good agreement between BIS and entropy (RE and SE).

Post-operatively, with the use of the Brice questionnaire, none of the patients reported awareness or unpleasant experiences. There were no perioperative complications.

\section{DISCUSSION}

Our study showed that both BIS and entropy performed well in patients with movement disorders, and the EEG-based DOA indices changed in a predictable manner. The indices showed a progressive reduction from induction until the surgical incision mirroring a gradual transition into deeper levels of anaesthesia and an increase during emergence from anaesthesia demonstrating the recovery of consciousness. All EEG indices showed substantially strong correlation with each other. Lack of correlation between EEG-based DOA indices with HR and MAP suggests that haemodynamic 


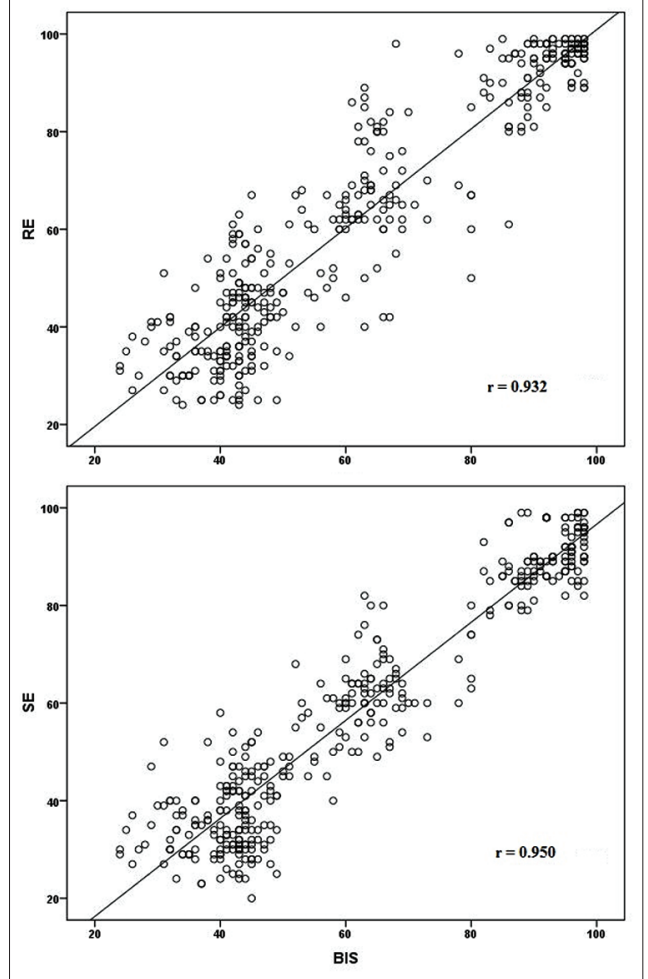

Figure 2: Linear regression curve of bispectral index versus response entropy and state entropy. Figure shows a strong correlation between bispectral index and response entropy, and also between bispectral index and state entropy

indices cannot be used alone as indicators of DOA in this patient population.

The use of EEG-based DOA monitors for titration of anaesthesia to minimize perioperative haemodynamic instability has been well established..$^{[5]}$ A recent Cochrane review has shown that anaesthesia guided by BIS improves anaesthetic delivery and recovery from anaesthesia. ${ }^{[23]}$ Another study showed that BIS-guided anaesthetic care also decreased post-operative delirium and cognitive decline and thus improved post-operative outcomes. ${ }^{[24]}$

Autonomic dysfunction is common in patients with Parkinson's disease and the maintenance of intra-operative haemodynamic stability under GA is often a challenge in these patients. Autonomic instability can lead to a sudden, exaggerated or unpredictable response to noxious stimuli and often leads to excessive administration of anaesthetics and analgesics. ${ }^{[25]}$ During internalisation of DBS, there are periods of intense stimulation (neck tunneling) in between long periods of minimal stimulation. In these fluctuating surgical conditions, maintaining a stable DOA based on intra-operative haemodynamics could be deceptive. The tendency to use minimal anaesthetics to avoid hypotension may increase the risk of awareness. These patients may also be at risk for post-operative delirium. ${ }^{[26]}$ The use of DOA monitors may be beneficial in providing

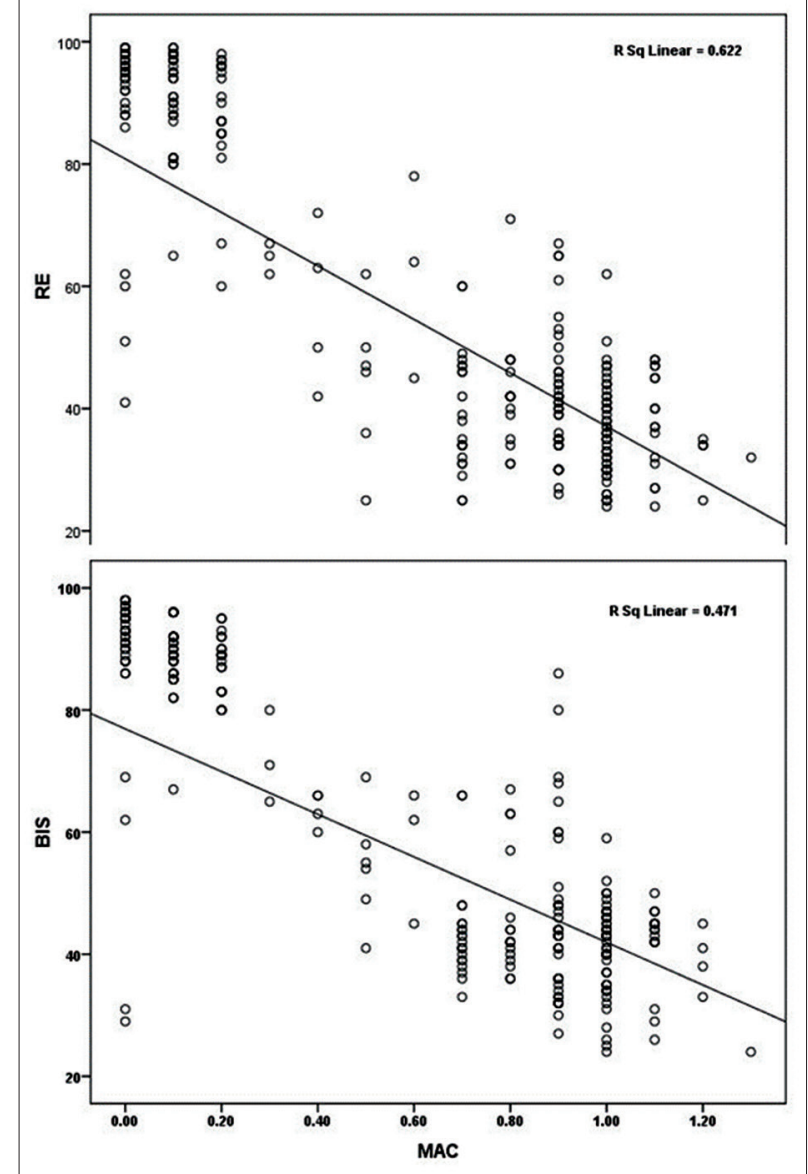

Figure 3: Linear regression curve of age-adjusted mean alveolar concentration versus bispectral index and response entropy. Figure shows a strong negative correlation between age-adjusted minimum alveolar concentration and bispectral index as well as with response entropy

guidance for the optimal delivery of anaesthetic agents to minimize perioperative haemodynamic instability and avoid post-operative complications in patients with movement disorders.

Originally, the calibrations of the EEG-based DOA monitors were performed on subjects with normal cerebral function. ${ }^{[10]}$ The presence of neurologic disorders that manifest with abnormal EEG patterns will, most likely, affect BIS monitoring. ${ }^{[20]}$ Entropy acts as its own control and has the potential benefit that it is independent of absolute values of the EEG frequency or amplitude, which are known to have inter-individual variation. ${ }^{[10]}$ There is limited data on the use of EEG-based DOA monitors in patients with neurological disorders. Pemberton and Dinsmore studied patients undergoing tumour or epilepsy surgery using a sleep-awake-sleep anaesthesia technique. ${ }^{[17]}$ They found a poor correlation between BIS values and the observer's assessment of anaesthesia level, suggesting that BIS is not a reliable tool for patients with brain abnormalities. However, a few recent studies have shown that there is a good correlation between both BIS and 
Table 4: Correlation analysis

\begin{tabular}{|c|c|c|c|c|c|}
\hline $\begin{array}{l}\text { Correlation } \\
\text { Indices }\end{array}$ & $\begin{array}{c}\text { Spearman } \\
\text { Correlation } \\
\text { coefficient (s) }\end{array}$ & $\begin{array}{c}\text { Pearson } \\
\text { Correlation } \\
\text { coefficient }(P)\end{array}$ & $\begin{array}{c}\text { Linear } \\
\text { regression } \\
\text { coefficient } r^{2}\end{array}$ & & $r P$ \\
\hline \multicolumn{6}{|l|}{ Variable: BIS } \\
\hline $\mathrm{RE}$ & 0.901 & 0.932 & 0.869 & 0.918 & 0.944 \\
\hline SE & 0.901 & 0.950 & 0.902 & 0.939 & 0.959 \\
\hline \multicolumn{6}{|c|}{ Variable: MAC } \\
\hline BIS & -0.620 & -0.686 & 0.471 & -0.735 & -0.630 \\
\hline $\mathrm{RE}$ & -0.726 & -0.788 & 0.622 & -0.823 & -0.748 \\
\hline $\mathrm{SE}$ & -0.677 & -0.732 & 0.536 & -0.775 & -0.683 \\
\hline \multicolumn{6}{|l|}{ Variable: HR } \\
\hline BIS & 0.158 & 0.190 & 0.036 & 0.092 & 0.284 \\
\hline $\mathrm{RE}$ & 0.200 & 0.198 & 0.039 & 0.101 & 0.291 \\
\hline $\mathrm{SE}$ & 0.201 & 0.204 & 0.041 & 0.107 & 0.297 \\
\hline \multicolumn{6}{|l|}{ Variable: MAP } \\
\hline BIS & 0.371 & 0.383 & 0.147 & 0.295 & 0.465 \\
\hline $\mathrm{RE}$ & 0.412 & 0.409 & 0.167 & 0.322 & 0.488 \\
\hline SE & 0.377 & 0.383 & 0.147 & 0.295 & 0.465 \\
\hline
\end{tabular}

$\mathrm{HR}=$ Heart rate, $\mathrm{MAP}=\mathrm{Mean}$ arterial concentration, aaMAC=Age adjusted, minimum alveolar concentration, $\mathrm{RE}=\mathrm{Response}$ entropy, $\mathrm{SE}=$ State entropy, BIS=Bispectral index

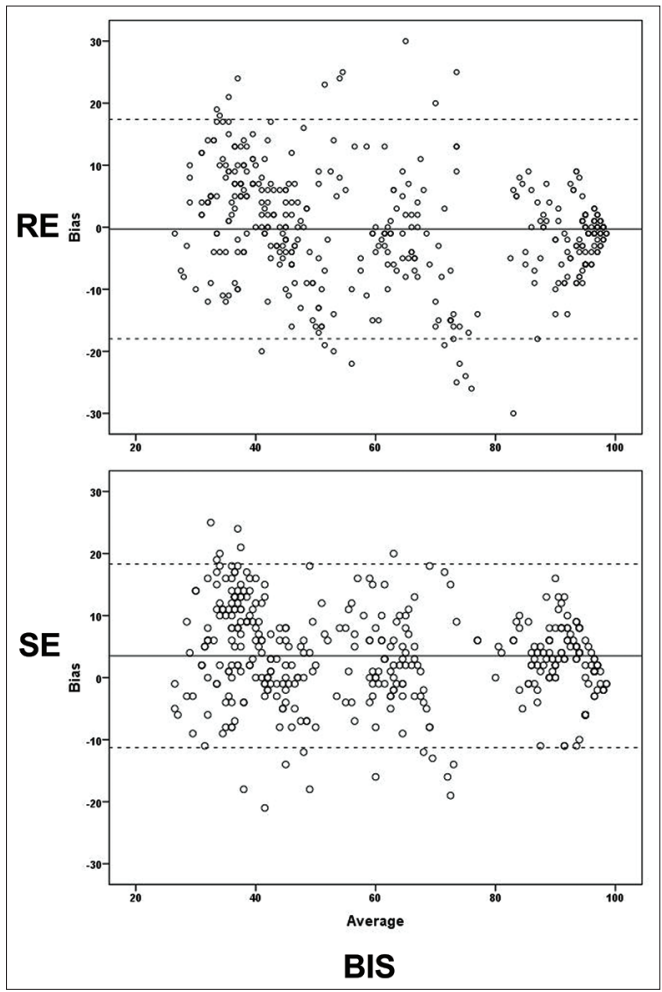

Figure 4: Bland-Altman plot of bispectral index and entropy. Figure shows a good agreement between bispectral index and both state entropy and response entropy. X-axis: Average of bispectral index and response entropy/state entropy respectively; Y-axis: Difference between bispectral index and response entropy/state entropy respectively. Nineteen data points were outliers in bispectral index versus response entropy and 15 data points were outliers in bispectral index versus state entropy entropy and clinical sedation scales in patients with brain lesions. ${ }^{[27]}$ Ozlu et al. found that BIS values correlated with the level of sedation in patients with Parkinson's disease undergoing DBS ${ }^{[19]}$ To our knowledge, there are no studies assessing the use of DOA monitors during GA in patients with movement disorders. Abnormalities in EEG have been reported in patients with Parkinson's disease, and the presence of pathological alpha dominant rhythm was seen in patients with significant cognitive fluctuations. ${ }^{[12]}$ This may limit the utility of EEG-based DOA monitors in these patients. However, sensors for the DOA monitor are usually placed in the front allocation and dominant (alpha) rhythm variations are mostly seen in the posterior brain regions. Hence, EEG-based DOA indices may not be affected, and our study does confirm this finding.

In our study, we found that correlation between BIS and entropy was good during various stages of surgery. BIS and RE were in agreement in all planes of anaesthesia and BIS and SE were in agreement mainly during surgical planes of anaesthesia as SE does not include the EMG component. However, we observed a large inter-individual variability in EEG indices. In the Bland-Altman analysis, the data points were widely scattered which probably indicates the pharmacological and physiological differences amongst the patients, making titration of anaesthesia by traditional haemodynamic markers less accurate. In addition, the aaMAC values in our study varied between 0.7 and 1.1 which might have contributed to the inter-individual variation in the EEG indices. 


\section{Limitation}

Our study does suffer from major limitations. First, this study is limited by a small sample size $(n=30)$ with different patient profiles. Our sample size was based on previous similar studies looking at the BIS and entropy in patients undergoing neurosurgical procedures. ${ }^{[21,22]}$ In addition, it is possible that the severity of disease might be different amongst the patients in our group which might have contributed to the some of the variations in the haemodynamic and EEG indices. Second, we did not know if any of our patients had preexisting baseline EEG changes. All patients had left sided placement of BIS and entropy sensors which may not reflect the underlying global picture. Hence, co-existing pathological EEG might not have affected the performance of BIS and entropy in this study. Finally, in our study, anaesthesiologists titrated the DOA on the basis of aaMAC values and clinical indices. As a result, there is a possibility of over or under dosing of anaesthetics which might have influenced the haemodynamic parameters.

\section{CONCLUSION}

Our study showed that both BIS and entropy performed well in patients with Parkinson's disease and other movement disorders. There was a good correlation between BIS and entropy devices. Haemodynamic parameters are not reliable indicators of DOA in this subset of patients probably due to pre-existing autonomic dysfunction.

\section{Financial support and sponsorship Nil.}

\section{Conflicts of interest}

There are no conflicts of interest.

\section{REFERENCES}

1. Palanca BJ, Mashour GA, Avidan MS. Processed electroencephalogram in depth of anesthesia monitoring. Curr Opin Anaesthesiol 2009;22:553-9.

2. Punjasawadwong Y, Boonjeungmonkol N, Phongchiewboon A. Bispectral index for improving anaesthetic delivery and postoperative recovery. Cochrane Database Syst Rev 2007; 17:CD003843.

3. Klopman MA, Sebel PS. Cost-effectiveness of bispectral index monitoring. Curr Opin Anaesthesiol 2011;24:177-81.

4. Monk TG. Processed EEG and patient outcome. Best Pract Res Clin Anaesthesiol 2006;20:221-8.

5. Johansen JW, Sebel PS, Sigl JC. Clinical impact of hypnotic-titration guidelines based on EEG bispectral index (BIS) monitoring during routine anesthetic care. J Clin Anesth 2000;12:433-43.

6. Smith D, Andrzejowski J, Smith A. Certainty and uncertainty: NICE guidance on 'depth of anaesthesia' monitoring. Anaesthesia 2013;68:1000-5.

7. Shepherd J, Jones J, Frampton G, Bryant J, Baxter L, Cooper K. Clinical effectiveness and cost-effectiveness of depth of anaesthesia monitoring (E-Entropy, Bispectral Index and Narcotrend): A systematic review and economic evaluation.
Health Technol Assess 2013;17:1-264.

8. Viertiö-Oja $H$, Maja $V$, Särkelä $M$, Talja $P$, Tenkanen $N$, Tolvanen-Laakso H, et al. Description of the Entropy algorithm as applied in the Datex-Ohmeda S/5 Entropy module. Acta Anaesthesiol Scand 2004;48:154-61.

9. Sebel PS, Bowdle TA, Ghoneim MM, Rampil IJ, Padilla RE, Gan TJ, et al. The incidence of awareness during anesthesia: A multicenter United States study. Anesth Analg 2004;99:833-9.

10. Arnold G, Kluger M, Voss L, Sleigh J. BIS and Entropy in the elderly. Anaesthesia 2007;62:907-12.

11. Fonseca LC, Tedrus GM, Letro GH, Bossoni AS. Dementia, mild cognitive impairment and quantitative EEG in patients with Parkinson's disease. Clin EEG Neurosci 2009;40:168-72.

12. Bonanni L, Thomas A, Tiraboschi P, Perfetti B, Varanese S, Onofrj M. EEG comparisons in early Alzheimer's disease, dementia with Lewy bodies and Parkinson's disease with dementia patients with a 2-year follow-up. Brain 2008;131 (Pt 3):690-705.

13. Venkatraghavan L, Luciano $M$, Manninen $P$. Review article: Anesthetic management of patients undergoing deep brain stimulator insertion. Anesth Analg 2010;110:1138-45.

14. Tallach RE, Ball DR, Jefferson P. Monitoring seizures with the bispectral index. Anaesthesia 2004;59:1033-4.

15. White PF, Rawal S, Recart A, Thornton L, Litle M, Stool L. Can the bispectral index be used to predict seizure time and awakening after electroconvulsive therapy? Anesth Analg 2003;96:1636-9.

16. Dahaba AA. Different conditions that could result in the bispectral index indicating an incorrect hypnotic state. Anesth Analg 2005;101:765-73.

17. Pemberton P, Dinsmore J. Bispectral index monitoring during awake craniotomy surgery. Anaesthesia 2002;43:708-10.

18. Renna M, Handy J, Shah A. Low baseline bispectral index of the electroencephalogram in patients with dementia. Anesth Analg 2003;96:1380-5.

19. Ozlu O, Sanalbas S, Yazicioglu D, Utebey G, Baran I. Sedation and regional anesthesia for deep brain stimulation in Parkinson's disease. J Anesthesiol 2014;2014:6.

20. Paolo Martorano P, Falzetti G, Pelaia P. Bispectral index and spectral Entropy in neuroanesthesia. J Neurosurg Anesthesiol 2006;18:205-10.

21. Moonesinghe SR, Walker EM, Bell M; SNAP - Investigator Group. Design and methodology of SNAP-1: A sprint national anaesthesia project to measure patient reported outcome after anaesthesia. Perioper Med (Lond) 2015;4:4.

22. Kaskinoro K, Maksimow A, Långsjö J, Aantaa R, Jääskeläinen S, Kaisti $\mathrm{K}$, et al. Wide inter-individual variability of bispectral index and spectral Entropy at loss of consciousness during increasing concentrations of dexmedetomidine, propofol, and sevoflurane. Br J Anaesth 2011;107:573-80.

23. Punjasawadwong $\mathrm{Y}$, Phongchiewboon A, Bunchungmongkol N. Bispectral index for improving anaesthetic delivery and postoperative recovery. Cochrane Database Syst Rev 2014;6:CD003843.

24. Chan MT, Cheng BC, Lee TM, Gin T; CODA Trial Group. BIS-guided anesthesia decreases postoperative delirium and cognitive decline. J Neurosurg Anesthesiol 2013;25:33-42.

25. Mustafa HI, Fessel JP, Barwise J, Shannon JR, Raj SR, Diedrich $\mathrm{A}$, et al. Dysautonomia: Perioperative implications. Anesthesiology 2012;116:205-15.

26. Strøm C, Rasmussen LS, Sieber FE. Should general anaesthesia be avoided in the elderly? Anaesthesia 2014;69 Suppl 1:35-44.

27. Deogaonkar A, Gupta R, DeGeorgia M, Sabharwal V, Gopakumaran B, Schubert A, et al. Bispectral index monitoring correlates with sedation scales in brain-injured patients. Crit Care Med 2004;32:2403-6. 


\section{APPENDIX 1}

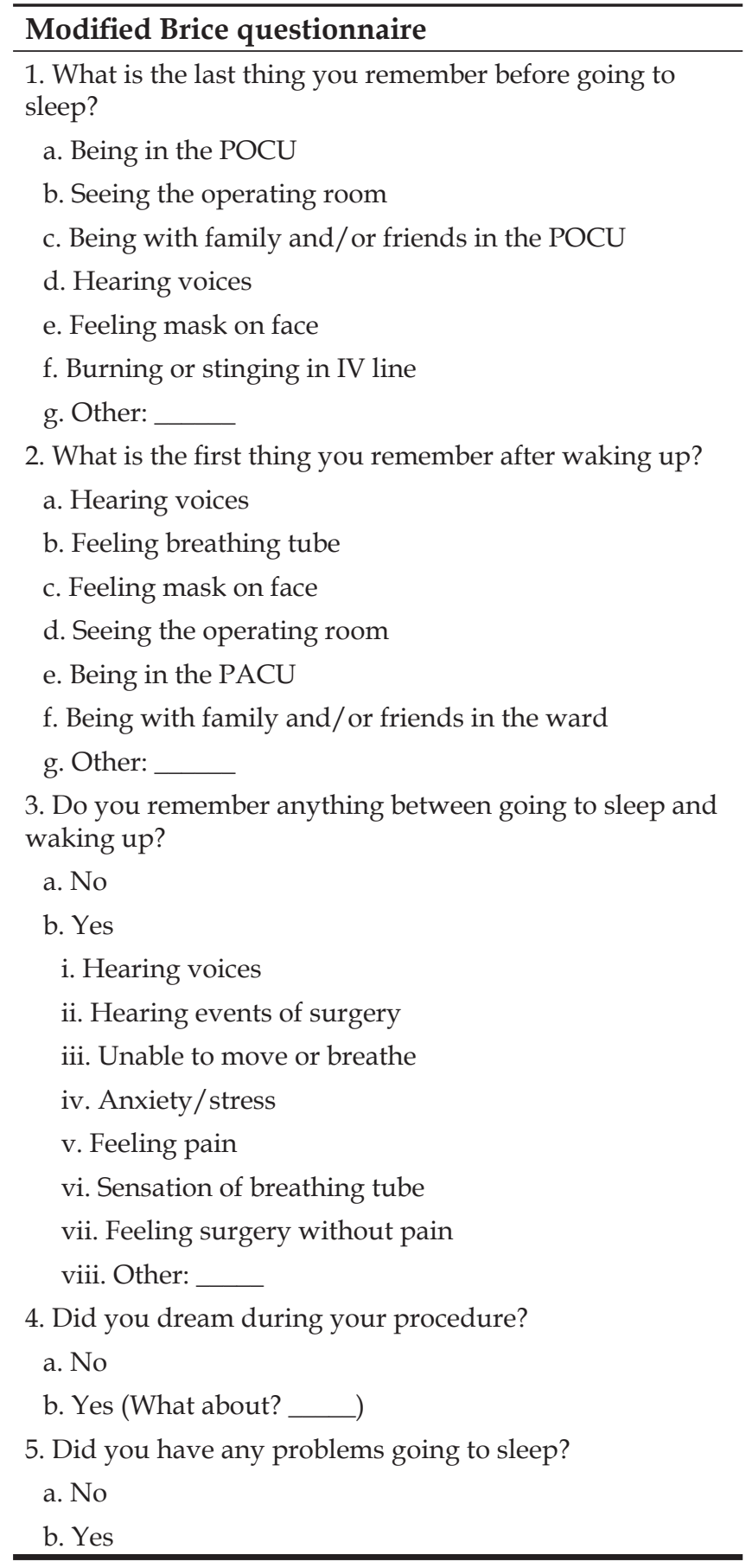

POCU: Pre-operative care unit, PACU: Post-anaesthesia care unit,

IV: Intravenous 